\title{
Substrate Wettability Influences Internal Jet Formation and Mixing during Droplet Coalescence
}

Thomas C. Sykes,* David Harbottle, Zinedine Khatir, Harvey M. Thompson, and Mark C. T. Wilson*

Cite This: Langmuir 2020, 36, 9596-9607

Read Online

ABSTRACT: The internal dynamics during the axisymmetric coalescence of an initially static free droplet and a sessile droplet of the same fluid are studied using both laboratory experiments and numerical simulations. A high-speed camera captured internal flows from the side, visualized by adding a dye to the free droplet. The numerical simulations employ the volume of fluid method, with the Kistler dynamic contact angle model to capture substrate wettability, quantitatively validated against the image-processed experiments. It is shown that an internal jet can be formed when capillary waves reflected from the contact line create a small tip with high curvature on top of the coalesced droplet that propels fluid toward

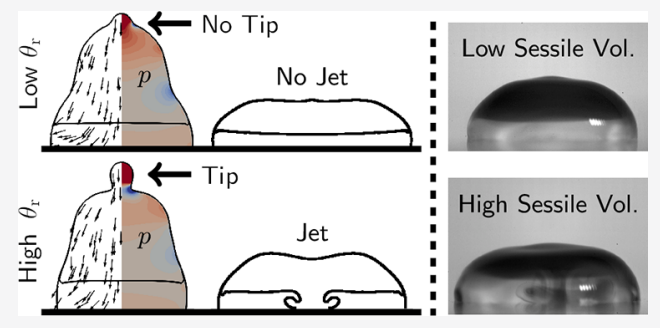
the substrate. Jet formation is found to depend on the substrate wettability, which influences capillary wave reflection; the importance of the advancing contact angle subordinated to that of the receding contact angle. It is systematically shown via regime maps that jet formation is enhanced by increasing the receding contact angle and by decreasing the droplet viscosity. Jets are seen at volume ratios very different from those accepted for free droplets, showing that a substrate with appropriate wettability can improve the efficiency of fluid mixing.

\section{INTRODUCTION}

Droplet coalescence on a substrate is a feature of many applications including inkjet printing, microfluidics, and coating. ${ }^{1,2}$ In many of these examples, effective mixing between the miscible fluids within each droplet is required, such as biochemical reagents and reactants in a range of microfluidic devices. ${ }^{3,4}$ Due to the short length scales (and often small velocities) involved, the internal flow generated by coalescence is typically laminar so effective mixing can be difficult to realize. ${ }^{5}$ Mixing can sometimes be enhanced by surface tension differences between coalescing droplets that induce Marangoni flows, which act on a short time scale, ${ }^{6,7}$ and concurrent chemical reactions can have a significant effect on coalescence behavior and internal flow. ${ }^{8}$ The presence of a substrate may influence mixing too. 9,10

A desirable way to improve advective mixing, and therefore the action of diffusion to homogenize a coalesced droplet, is by forming an internal jet that stretches and folds the internal fluid interface. Internal jets in coalescing droplets not in contact with a substrate (free droplets) have been studied extensively. Given a sufficient size ratio between droplets of the same fluid, the difference in Laplace pressure can cause the smaller droplet to be injected into the larger one in the form of a jet. ${ }^{11,12}$ Note that jets cannot occur during the coalescence of identical, static free droplets by symmetry. ${ }^{3}$ The kinetic energy of internal jets is inherited from excess free surface energy so jet formation relies on the flow being dominated by surface tension. ${ }^{13}$ Given a sufficient increase in droplet viscosity (characterized by the Ohnesorge number), enough surface energy can be eliminated by viscous dissipation that the smaller droplet is simply lodged into the larger droplet with little advective mixing. ${ }^{14}$ Recently, jet formation in free droplets has been associated with the formation of a vortex ring, where growth and eventual detachment are both required, after which the vortex ring must have sufficient momentum to overcome viscous dissipation for a jet to form. ${ }^{15}$

Internal jets can be seen in other cases too, such as colliding droplets where jet emergence has a nontrivial relationship with velocity. ${ }^{16}$ Another example is droplet-pool coalescence (the limit of large size ratio) for both slowly coalescing and impacting droplets. ${ }^{17}$ In these cases, jets may be enhanced by surface tension gradients due to Marangoni flow and the influence of surface tension on Laplace pressure. ${ }^{18,19}$ Hence, a size difference between coalescing droplets of different fluids is not necessarily required for jet formation.

There is limited study of internal jet formation in the presence of a substrate, though a jet can be formed during the coalescence of an impacting and a sessile droplet if the latter is much larger. ${ }^{20}$ When a substrate is involved, there is the added aspect of a three-phase contact line which can affect the flow. ${ }^{21}$ Several studies have considered internal flows within coalescing sessile droplets on a substrate with a wettability

Received: June 8, 2020

Revised: July 20, 2020

Published: August 6, 2020 
gradient, which can enhance mixing. ${ }^{20}$ Motion of the contact line during coalescence has been shown to be beneficial for mixing by inducing jet-like horizontal recirculatory flow for both identical and distinct fluids. ${ }^{4,10}$ However, a recent study has indicated that mixing is not enhanced by a wettability gradient for simultaneously deposited droplets with nonzero lateral separation. ${ }^{22}$

Dynamic contact angles also play a role in mixing on substrates with uniform wettability. Internal flows have been considered between impacting droplets with varying lateral separation and (fixed) nonzero hysteresis, but little mixing was observed. $^{23}$ For initially sessile, sliding droplets on superhydrophobic substrates, low-contact-angle hysteresis enhances the rate and extent of mixing due to increased droplet oscillation and deformation compared to high hysteresis substrates. ${ }^{9}$ Though dynamic contact angles have recently been shown to be important in determining droplet dynamics during splashing, ${ }^{24}$ there is a lack of a systematic study of their effect on mixing during coalescence.

In this work, the influence of substrate wettability and dynamic contact angles on internal jet formation during the coalescence of a free droplet and a sessile droplet is systematically studied for the first time, using both highspeed imaging and numerical simulations. Wettability is accurately captured in the simulations through the Kistler dynamic contact angle model (including hysteresis), which allows the limiting advancing and receding contact angles to be independently varied. In addition, the experimental procedure ensures that the droplets are dominated by surface tension and have negligible velocity at the onset of coalescence. Hence, the influence of capillary waves reflected from the contact line on the internal flow and jet formation are determined in tandem with the effect of the volume ratio and droplet fluid properties. Throughout, careful comparison to the more widely studied free droplet coalescence is made to elucidate the differences caused by the substrate.

\section{EXPERIMENTAL DETAILS}

Materials and Characterization. All droplets consisted of ultrapure Milli-Q water with a surface tension of $72.4 \pm 0.2 \mathrm{mN}$ $\mathrm{m}^{-1}$, measured by a pendant droplet tensiometer (Biolin Scientific Theta T200) analyzing a $13 \mu \mathrm{L}$ droplet at the end of a stainless steel blunt end dispensing tip (Fisnar 22 gauge). Nigrosin water soluble dye (Alfa Aesar) was added to the free droplet at $0.3 \mathrm{~g} \mathrm{~L}^{-1}(300 \mathrm{ppm})$ to visualize the internal flow. The surface tension of the dyed fluid was verified to be the same as the undyed fluid (over $120 \mathrm{~s}$, repeated four times), within experimental error, so both dyed and undyed fluids have identical fluid properties. The density and dynamic viscosity were $997 \pm 1 \mathrm{~kg} \mathrm{~m}^{-3}$ and $0.93 \pm 0.01 \mathrm{mPa}$ s, respectively.

Coalescence occurred between a free droplet and a sessile droplet on a silanised glass slide (Fisherbrand plain glass, thickness 1.0-1.2 $\mathrm{mm}$ ). To prepare each substrate, a new slide was rinsed with Milli- $Q$ water and dried with nitrogen before being exposed to $0.5 \mathrm{~mL}$ of a silane solution (dichloromethyl- $n$-octylsilane, 98\%, Alfa Aesar) in a sealed container to allow vapor deposition for 6-8 min. The slide was subsequently rinsed and air-dried prior to use. The droplets were quickly removed by a shear air flow following each experiment with no discernible change in wettability within a given area. The substrate properties (including wettability) are examined later.

Procedure. The experimental setup is depicted in Figure 1. The sessile droplet was manually deposited onto the substrate using either a 26 gauge needle syringe (Hamilton GASTIGHT $1701 \mathrm{~N}, 10 \mu \mathrm{L}$ ) or a variable volume pipette (Thermo Scientific Finnpipette F2, 10-100 $\mu \mathrm{L}$ ), depending on the desired volume. The substrate was elevated by $5 \mathrm{~mm}$ acrylic blocks above an aluminum foil-covered translation stage

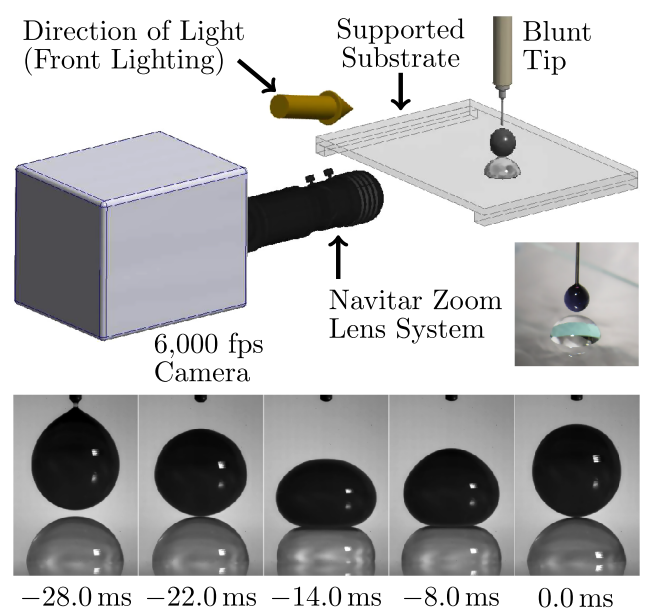

Figure 1. Schematic diagram of the experimental setup, with an inset real image from the same view point. The lower image montage illustrates the bouncing dynamics, which generate the static initial condition.

(providing two-axis horizontal motion) to improve illumination. The free droplet was generated by dripping from a stainless steel blunt end dispensing tip (Fisnar 30 gauge), mounted on a vertical motorized stage, attached to an automated syringe pump as part of the tensiometer.

In each experiment, the sessile droplet was deposited while a stable pendant droplet formed. The sessile droplet was conveyed by the translation stage to ensure axisymmetry of the pendant and sessile droplets, monitored by a high-speed camera and an alignment camera as part of the tensiometer, positioned perpendicular to each other. Further fluid was then injected into the pendant droplet at $0.1 \mu \mathrm{L} \mathrm{s}^{-1}$ until it detached due to gravity and fell vertically toward the sessile droplet. At the point of detachment, the droplet free surfaces were $0.17 \pm 0.01 \mathrm{~mm}$ apart. The volume of the free droplet, $V_{\mathrm{f}}$, was measured by the tensiometer via an edge-detection routine as $6.2 \mu \mathrm{L}$ in all experiments. The sessile droplet volume, $V_{s}$, was determined by image processing the high-speed camera images to nullify any effects of evaporation or uncertainty in deposition $( \pm 0.1 \mu \mathrm{L})$.

The image montage within Figure 1 shows that coalescence did not occur immediately after the free droplet landed. Instead, the free droplet slowly bounced on an air layer that was sufficiently thick to prevent intermolecular (van der Waals) forces from causing it to instantly rupture, delaying coalescence. ${ }^{25}$ Viscous dissipation reduced the minimal initial kinetic energy during this time. After one bounce, the high surface tension recovered the original spherical droplet shape and coalescence was initiated at the point of separation, which defines the initial condition for coalescence. ${ }^{26}$ The relative droplet velocity was estimated to be $\ll 5 \times 10^{-2} \mathrm{~mm} \mathrm{~ms}^{-1}$ at this time, which is sufficiently small that any remaining kinetic energy was dominated by meniscus bridge growth post coalescence. ${ }^{27}$ Hence, the initial condition is effectively two static droplets, where the free droplet was not attached to any structure, which would influence the ensuing intricate free surface dynamics. Time zero $(t=0)$ is taken as the frame immediately before the air layer visibly ruptured. Each experiment was repeated at least five times to establish the typical dynamics that are reported. All experiments took place at room temperature $(23 \pm 2$ ${ }^{\circ} \mathrm{C}$ ) and atmospheric pressure.

The ratio of viscous to inertial and surface tension forces is characterized by the Ohnesorge number, Oh $=\mu_{\mathrm{d}} / \sqrt{\rho_{\mathrm{d}} \sigma r_{\mathrm{f}}}$, where $\mu_{\mathrm{d}}$, $\rho_{\mathrm{d}}$, and $\sigma$ are the droplet dynamic viscosity, density, and surface tension, respectively. The length scale is chosen as the free droplet radius, $r_{\mathrm{f}} \approx 1.14 \mathrm{~mm}$ so $\mathrm{Oh} \approx 3.2 \times 10^{-3}$, indicating that the flow is dominated by surface tension. Furthermore, the Bond number is Bo = $g r_{\mathrm{f}}^{2} \Delta \rho / \sigma \approx 0.18$, where $g$ is the gravitational acceleration and $\Delta \rho \approx$ $10^{3} \mathrm{~kg} \mathrm{~m}^{-3}$ is the density difference between the droplets and surrounding air. It was observed that the sessile droplet forms a 
spherical cap for moderate volumes $(\leq 10 \mu \mathrm{L})$, confirming that surface tension dominates gravitational forces.

Imaging. A single high-speed camera (a monochrome Photron FASTCAM SA5 775K-M3) captured the dynamics from the side. A zoom lens system was used, consisting of body (Navitar 12X Zoom, 150486) and adapter (Navitar 2X F-mount, 1-62922) tubes, with no lens attachment, yielding a working distance of $86 \mathrm{~mm}$. The pixel resolution was $1024 \times 768$, giving an effective resolution of $134.5 \pm$ 1.5 pixels $\mathrm{mm}^{-1}$, which was sufficient to accurately measure the apparent contact angles. The camera was inclined downward approximately $2^{\circ}$ relative to the substrate to reduce glare around the free surface. The images were recorded at 6000 frames per second (fps), with an exposure of approximately $143 \mu \mathrm{s}$.

The droplets were front-lit by a single cold white $84 \mathrm{~W}$ LED light (MultiLED LT) positioned above and to the right of the zoom lens to maximize brightness and minimize reflections. A white background, in addition to the previously discussed substrate position, was crucial for enabling the use of a sufficiently short exposure. The light was only switched on for a short and consistent time (approximately $10 \mathrm{~s}$ ) during each experiment.

\section{NUMERICAL SIMULATIONS}

Mathematical Model. The volume of fluid (VOF) diffuseinterface method is used to capture the free surface dynamics, discretised by the finite volume method and implemented within an open-source numerical toolbox (OpenFOAM). ${ }^{28}$ The volume fraction, $\alpha \in[0,1]$, a conserved scalar, identifies all droplets (where $\alpha=1$ ) within the air (where $\alpha=0$ ). Both the droplet and air phases can therefore be modeled as a single fluid, with combined (mixture) fluid properties. The mixture density is a volume-fraction-weighted average of the density of each fluid phase given by $\rho=\alpha \rho_{\mathrm{d}}+(1-\alpha) \rho_{\mathrm{o}}$, where $\rho_{\mathrm{d}}$ and $\rho_{\mathrm{o}}$ are the droplet and air densities, respectively. Similarly, the mixture dynamic viscosity is $\mu=\alpha \mu_{\mathrm{d}}+(1-\alpha) \mu_{\mathrm{o}}$, where $\mu_{\mathrm{d}}$ and $\mu_{\mathrm{o}}$ are the droplet and air dynamic viscosities, respectively.

A single set of Navier-Stokes equations is solved for the combined Newtonian fluid. Hence, the continuity equation is

$$
\frac{\partial \rho}{\partial t}+\frac{\partial}{\partial x_{j}}\left(\rho u_{j}\right)=0
$$

where $u_{j}$ is the fluid velocity and summation over repeated indices is implied. The momentum equation reads

$$
\frac{\partial}{\partial t}\left(\rho u_{i}\right)+\frac{\partial}{\partial x_{j}}\left(\rho u_{i} u_{j}\right)=-\frac{\partial p}{\partial x_{i}}+\frac{\partial}{\partial x_{j}}\left(\mu\left(\frac{\partial u_{i}}{\partial x_{j}}+\frac{\partial u_{j}}{\partial x_{i}}\right)\right)+F_{i}
$$

where $p$ is the pressure and $F_{i}$ represents any body forces.

Surface tension is included through the Brackbill continuum surface force model by approximation as a body force near the free surface, identified by the volume fraction. ${ }^{29}$ Hence,

$$
F_{i}=\sigma \kappa \frac{\partial \alpha}{\partial x_{i}}
$$

where $\sigma$ is the surface tension and $\kappa$ is the free surface curvature, given by

$$
\kappa=-\frac{\partial}{\partial x_{j}}\left[\frac{\partial \alpha}{\partial x_{j}} /\left(\frac{\partial \alpha}{\partial x_{k}} \frac{\partial \alpha}{\partial x_{k}}\right)^{1 / 2}\right]
$$

where the quantity in the square brackets represents the unit normal to the free surface. The normal is therefore computed algebraically from the volume fraction, rather than a geometric reconstruction of the free surface. Simulations are restricted to cases where gravity is negligible (Bo $\lesssim 0.2$ ) so gravity is not included in eq 3 .

The volume fraction is advected with the updated velocity every time step according to

$$
\frac{\partial \alpha}{\partial t}+\frac{\partial}{\partial x_{j}}\left(\alpha u_{j}\right)+\frac{\partial}{\partial x_{j}}\left(u_{c, j} \alpha(1-\alpha)\right)=0
$$

where $u_{c, j}$ is a compression velocity. The last term on the lefthand side of eq 5 is present solely to yield a sharp free surface by limiting numerical diffusion associated with advection of a (analytical) step function. It is only nonzero in the region of the free surface. ${ }^{30}$ The coefficient of the compression term is taken to be unity; further technical details are given in the Supporting Information. Molecular diffusion is not included in eq 5 due to the size of the droplets (millimetric) and short time scales (millisecond) considered.

To enable the internal dynamics and extent of advective mixing to be determined, an additional conserved scalar, $\beta \in$ $[0,1]$ is added, where $\beta=1$ only within the free droplet and $\beta$ $=0$ elsewhere. $\beta$ is advected in the same way as $\alpha$ by an advection-diffusion equation equivalent to eq 5. Unlike $\alpha$ though, there is a one-way coupling between $\beta$ and the flow (eqs 1 and 2), so $\beta$ is only a passive scalar, identifying the fluid from the free droplet (where $\beta=1$ ) within the entire coalesced droplet (where $\alpha=1$ ) while having no influence on the dynamics.

Model Substrate. The use of an appropriate dynamic contact angle model to relate contact line velocity to the apparent dynamic contact angle is essential to accurately capture substrate wettability. A review of such models can be found elsewhere, ${ }^{31}$ but this work uses the empirical correlation given by Kistler, $^{32}$ originally derived by fitting data of advancing liquid-air free surfaces in capillaries. ${ }^{33}$ The Kistler model has been used in various previous studies of droplet dynamics $^{34-36}$ and is appropriate for this work since the contact line only undergoes perturbations from an equilibrium state (of the sessile droplet) on a partially wetted substrate instead of being formed, the latter being the case for impact onto dry substrates where $\theta=180^{\circ}$ initially. The dynamic contact angle $\theta$ is given by

$$
\theta=f_{\mathrm{H}}\left[\mathrm{Ca}+f_{\mathrm{H}}^{-1}(\Theta)\right]
$$

where $\mathrm{Ca}$ is the contact line capillary number and $f_{\mathrm{H}}$ is the Hoffman function,

$$
f_{\mathrm{H}}(s)=\arccos \left\{1-2 \tanh \left[5.16\left(\frac{s}{1+1.31 s^{0.99}}\right)^{0.706}\right]\right\}
$$

The contact line capillary number is given by $\mathrm{Ca}=\mu \mid u_{\mathrm{cl}} \mathrm{l} / \sigma$, where $u_{\mathrm{cl}}$ is the contact line speed determined from the center of the cell closest to the substrate at the contact line (characterized by $\alpha$ ) since OpenFOAM uses a collocated methodology. To capture contact angle hysteresis, $\Theta$ is a dummy variable in eq 6 whose value depends on the direction of the contact line motion via

$$
\Theta= \begin{cases}\theta_{\mathrm{a}} & \text { for } u_{\mathrm{cl}}>0 \\ \theta_{0} & \text { for } u_{\mathrm{cl}}=0 \\ \theta_{\mathrm{r}} & \text { for } u_{\mathrm{cl}}<0\end{cases}
$$


where $\theta_{a}, \theta_{0}$, and $\theta_{\mathrm{r}}$ are the prescribed (minimum) advancing, equilibrium, and (maximum) receding contact angles, respectively. ${ }^{35}$ These prescribed values are independently varied to alter the substrate wettability $\left(\theta\right.$ for a given $\left.u_{\mathrm{cl}}\right)$ in this work. This dynamic contact angle model has been implemented in OpenFOAM ${ }^{37,38}$ and is quantitatively validated against experimental data in Figure 2.

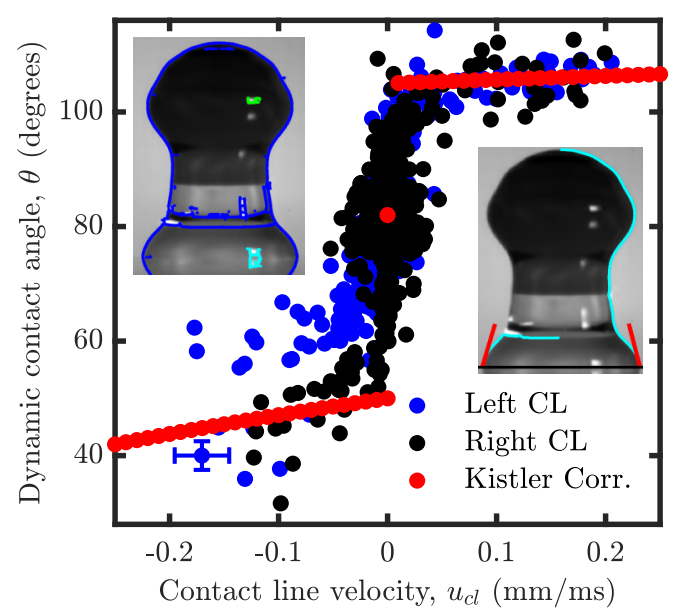

Figure 2. Image-processed contact angles against contact line velocity, the latter approximated from the evolution of the spread length. The inset images show detected edges and tangents to contact lines at $t=$ $2.0 \mathrm{~ms}$. The error bars are omitted from all but one point (bottom left).

Equation 6 prescribes the orientation of the free surface near the substrate, where it is enforced as a boundary condition on $\alpha$ (and $\beta$ ) by updating the normal in eq 3 on each time step. ${ }^{39}$ No-slip is applied on the substrate (cell faces) and the pressure boundary condition is chosen to be determined by the velocity (fixedFluxPressure in OpenFOAM).

Geometry. All simulations reported are axisymmetric so were performed in a quarter domain, with symmetry planes on the inner boundaries, to reduce the computational cost. The mesh consisted of hexahedral cells and is adaptively refined only where $\alpha>0.01$ every two time steps. The dynamics were found to be remarkably insensitive to the mesh resolution within the air, so a base mesh of 10 cells per free droplet radius $r_{\mathrm{f}}$ was employed. A relatively fine mesh was required within the droplet so 3 levels of refinement (i.e., a multiple of $2^{3}$ ) were deployed, yielding a mesh resolution of 80 cells per $r_{\mathrm{f}}$ within the whole droplet volume. Despite the diffuse interface, further refinement had a negligible effect on the results. With around $1.1 \times 10^{6}$ cells, all free surface and internal features were sufficiently resolved (see the Supporting Information for a mesh independence study). The initial $\alpha$ and $\beta$ distributions were defined on the refined mesh.

The sessile droplet is assumed to be a spherical cap, defined by its volume $V_{s}$ and equilibrium contact angle $\theta_{0}$. For comparison to the experimental data, the spread length and equilibrium contact angle were matched to determine $V_{s}$. Free droplets are spherical with volume $V_{\mathrm{f}}$. The meniscus bridge inevitably had a finite width of $<0.2 r_{\mathrm{f}} \mathrm{mm}$ (on each side) at $t$ $=0$, modified to prevent air entrapment during meniscus bridge expansion as necessary without affecting the capillary waves. ${ }^{15}$ The domain was initialized with uniform zero pressure and velocity. It has been checked that the solver correctly recovers the analytical Laplace pressure of a free droplet within $5 \times 10^{-3}$ capillary time units, which is approximately $23 \mu \mathrm{s}$ for $V_{\mathrm{f}}=6.2 \mu \mathrm{L}$ (see the Supporting Information).

The dynamics were found to be very insensitive to the domain size; $4 r_{\mathrm{f}} \times 5 r_{\mathrm{f}} \times 4 r_{\mathrm{f}}$ was chosen for the quarter domain. Thus, the atmospheric (open) boundaries remain sufficiently far from the droplets that their boundary conditions have a negligible effect on the dynamics. Zero Neumann and Dirichlet boundary conditions were applied there for velocity and modified pressure, respectively. ${ }^{39,40}$ The volume fraction and passive scalar obey zero Neumann and Dirichlet boundary conditions for outflow and inflow, respectively, which ensures that only air can enter the domain. ${ }^{41}$

Implementation. Simulations were carried out using OpenFOAM (Foundation) 4.1, with a version of the interFoam solver augmented by the modifications described above. Details of the scalar transport, discretisation schemes, and linear solvers are provided in the Supporting Information.

The prescription of a small initial time step was crucial for maintaining interface stability, set as $1 \mathrm{~ns}$ in all simulations. Thereafter, the time step was varied while respecting a maximum Courant number of 0.15 , with a typical time step around $1 \mu \mathrm{s}$. A typical simulation from $t=0$ to $20 \mathrm{~ms}$ required approximately 300 core hours, with a wall clock time around $23 \mathrm{~h}$, using 16 cores on a single node (of the ARC4 CentOS 7 cluster at the University of Leeds) containing Intel Xeon Gold 6138 ("Skylake") processors.

Unless stated otherwise, the simulations reported are of water droplets in air at $23{ }^{\circ} \mathrm{C}$, consistent with the experiments. The droplets have a density of $\rho_{\mathrm{d}}=997 \mathrm{~kg} \mathrm{~m}^{-3}$ and a kinematic viscosity of $\nu_{\mathrm{d}}=9.3 \times 10^{-7} \mathrm{~m}^{2} \mathrm{~s}^{-1}$ (as specified in OpenFOAM), corresponding to a dynamic viscosity of $\mu_{\mathrm{d}}=$ $9.3 \times 10^{-4} \mathrm{~Pa}$. The air has a density of $\rho_{\mathrm{o}}=1.2 \mathrm{~kg} \mathrm{~m}^{-3}$ and a kinematic viscosity of $\nu_{\mathrm{o}}=1.5 \times 10^{-5} \mathrm{~m}^{2} \mathrm{~s}^{-1}$, corresponding to a dynamic viscosity of $\mu_{\mathrm{o}}=1.8 \times 10^{-5} \mathrm{~Pa} \mathrm{~s}$.

\section{RESULTS AND DISCUSSION}

Substrate Wettability. To assess the substrate wettability, the contact angles were extracted from a typical experiment by image processing and are plotted in Figure 2 against contact line velocity, the latter approximated by forward differencing the spread length at the temporal resolution of the camera. The images were processed using a monochrome variant of an existing protocol, ${ }^{7}$ while the contact angles were determined using a recent method without the fitting of the analytical curves. $^{42}$ An example of a processed frame (at $t=2.0 \mathrm{~ms}$, where the left and right contact angles are $73.5 \pm 2.0^{\circ}$ and 75.5 $\pm 2.0^{\circ}$, respectively) is inset in Figure 2 . The left image shows the initial detected edges; the right image, filtered edges. The estimated error in the measurement of the dynamic contact angle is $\pm 2.0^{\circ}$ (combining systematic and random errors of $\pm 1.0^{\circ}$ each), while that of $u_{\mathrm{cl}}$ is $\pm 0.03 \mathrm{~mm} \mathrm{~ms}^{-1}$ based on the spread length and time resolution. The dispersion of the points is otherwise a reflection of variability. The red lines represent the detected tangents at the left and right contact points, from which the contact angles are determined.

Figure 2 shows that the substrate has a hysteresis of $\theta_{\mathrm{a}}-\theta_{\mathrm{r}}$ $\approx 50^{\circ}$, with $\theta_{\mathrm{a}} \approx 105^{\circ}$. With regard to the receding contact angle, the substrate is best described as being prone to pinning. Hence, a wider range of receding contact angles are seen in Figure 2. It should also be noted that the right contact angle is more amenable to image processing, due to the requisite light position, which accounts for the discrepancy between the left 

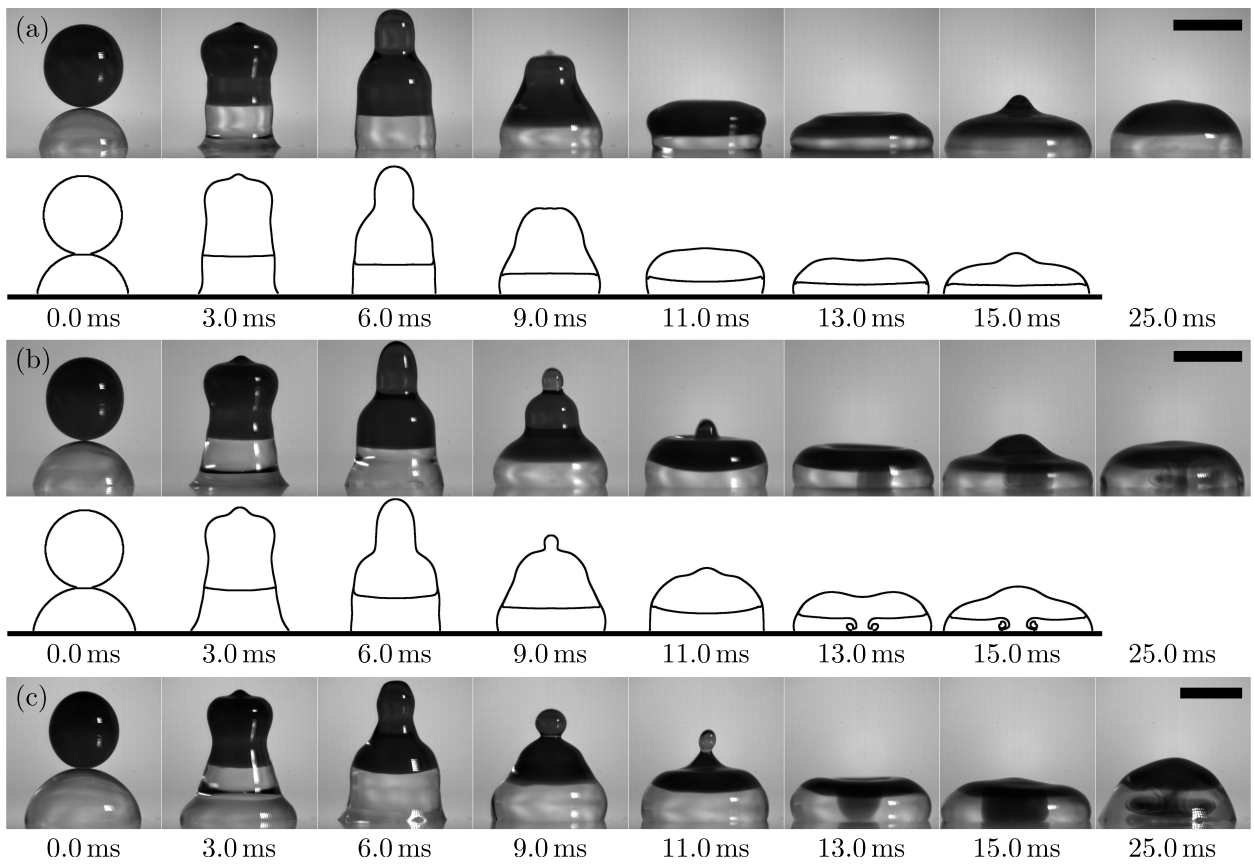

Figure 3. Side views of coalescence between a dyed free droplet and undyed sessile droplet of the same fluid. Free surface and internal interface profiles are shown for the simulations. The free droplet volume is fixed $\left(V_{\mathrm{f}}=6.2 \mu \mathrm{L}\right)$, while the sessile droplet volume is varied between the panels: (a) $V_{\mathrm{s}}=3.9 \mu \mathrm{L}$, with little advective mixing $\left(\theta_{0} \approx 82^{\circ}\right)$; (b) $V_{\mathrm{s}}=5.5 \mu \mathrm{L}$, with an internal jet $\left(\theta_{0} \approx 80^{\circ}\right)$; and (c) $V_{\mathrm{s}}=11.0 \mu \mathrm{L}$, with an internal jet. For the simulations, $\theta_{\mathrm{a}}=105^{\circ}$ and $\theta_{\mathrm{r}}=50^{\circ}$ are prescribed. All scale bars are $2 \mathrm{~mm}$.

and right receding contact angles; both the contact angle measurement and perceived contact line position are affected by this.

The Kistler model used in the numerical simulations (defined by eqs $6-8$ ) is also shown in Figure 2 with $\theta_{\mathrm{a}}=$ $105^{\circ}, \theta_{0}=82^{\circ}$, and $\theta_{\mathrm{r}}=50^{\circ}$ prescribed. Excellent agreement with the experimental data is evident, confirming the applicability of the Kistler model and the underlying contact angles prescribed. The only appreciable limitation of the Kistler model in the droplet configuration studied is that it does not include pinning as the contact line velocity determines the contact angle; the lack of pinning turns out to be inconsequential as elucidated when making a quantitative comparison between the experiments and simulations.

Qualitative Analysis. Figure 3 shows a montage of experiments and simulations for three different sessile droplet volumes, with a fixed free droplet volume of $V_{\mathrm{f}}=6.2 \mu \mathrm{L}$. In each case, rapid widening of the meniscus bridge between the coalescing droplets generates capillary waves due to the high curvature there. These capillary waves propagate outward along the free surface, both toward the top of the coalesced droplet and toward the substrate. The upward traveling capillary waves converge at the top of the droplet (visible at $t=3.0 \mathrm{~ms}$ ), where they stretch the free surface upward to form a tall cylindrical column (visible at $t=6.0 \mathrm{~ms}$ ). Due to the excess surface energy and higher pressure acquired, the column subsequently collapses toward the substrate to form a sessile droplet of lower surface energy. ${ }^{15}$ Free surface oscillations are initially large due to the substantial conversion of surface energy to kinetic energy but subsequently reduce due to viscous dissipation.

The dye added to the free droplet in the experiments allows the internal dynamics to be visualized. In Figure 3a, where $V_{\mathrm{s}}=$ $3.9 \mu \mathrm{L}$, the coalesced droplet flattens after the column collapses to a pancake shape (visible at $t=13.0 \mathrm{~ms}$ ) at its maximum spread length, but subsequently recoils to reveal a flat internal interface at $t=25.0 \mathrm{~ms}$. Any advective mixing is insignificant so the subsequent diffusive mixing stage required to homogenize the coalesced droplet could be on the order of minutes. ${ }^{7}$ However, on increasing the sessile droplet volume to $V_{\mathrm{s}}=5.5 \mu \mathrm{L}$ (Figure $3 \mathrm{~b}$ ), a vertical flow of dyed fluid into the undyed fluid below is visible at $t=13.0 \mathrm{~ms}$, which quickly develops into an internal jet. The presence of the jet improves advective mixing by stretching and folding the internal fluid interface $^{20}$ and is maintained on increasing the sessile droplet volume to $V_{\mathrm{s}}=11.0 \mu \mathrm{L}$ in Figure $3 \mathrm{c}$.

In Figure $3 b$, the larger volume droplet is injected into the other to form a jet, which is opposite to the typical situation in free droplet coalescence. ${ }^{11}$ Indeed, the sessile droplet has a lower curvature (and smaller Laplace pressure) at the onset of coalescence than if the substrate were not present, for the fixed volume. Furthermore, the jets seen in Figure 3 do not appear to be of the vortex ring kind seen in free droplets, ${ }^{15}$ but are instead reminiscent of an impinging jet with the substrate redirecting the downward flow to drive recirculation. The presence of the substrate may therefore enhance jet formation, potentially enabling good advective mixing for smaller droplet volumes than would otherwise be expected.

There are several contributory factors to jet formation in Figure 3. With an increase in sessile droplet volume, the curvature between the droplets at the onset of coalescence is greater leading to stronger capillary waves. ${ }^{40}$ Hence, greater stretching of the coalesced droplet is observed with increasing sessile droplet volume leading to a larger excess surface energy, which can be translated into kinetic energy to form a jet. Sufficient vertical space is also required in the undyed fluid for a jet to form; this space is of course expanded by increasing the sessile droplet volume. Importantly, the high substrate hysteresis also delays outward spreading of the droplet during column collapse, which increases the height of the internal 
interface within the coalesced droplet. Separately, wettability also affects the interaction between the substrate and the downward propagating capillary waves, which may affect jet formation. These factors can be analyzed using numerical simulations.

The simulation results displaying both the free surface (defined by $\alpha=0.2$ ) and internal interface (defined by $\beta=0.2$ ) profiles are shown in Figure 3 for the two smaller sessile droplet volumes where gravity is certainly negligible. The prescribed advancing and receding contact angles $\left(\theta_{\mathrm{a}}=105^{\circ}\right.$, $\theta_{\mathrm{r}}=50^{\circ}$ ) are consistent with Figure 2. To match the pertinent experiments, $\theta_{0}=82^{\circ}$ in Figure $3 \mathrm{a}$ and $\theta_{0}=80^{\circ}$ in Figure $3 \mathrm{~b}$. Note that the simulation results are represented by a cut-plane through the axis of symmetry, whereas the experiments adopt an external side view so depressions in the free surface are not conspicuous in the experimental images (e.g., at $t=13.0 \mathrm{~ms}$ in Figure $3 \mathrm{~b}$ ). Based on a qualitative assessment, the simulations are in excellent agreement with the experiments. All of the main features are captured accordingly, including the column stretching and subsequent collapse. Importantly, the simulations correctly predict the formation of a jet only where one is seen experimentally (i.e., Figure $3 b$ ). Of special note are the free surface shapes near the contact lines where excellent agreement between the experiments and simulations is evident.

Quantitative Assessment. Further image processing was undertaken on the experiments shown in Figure 3 to extract the spread length, $s$, total droplet height, and internal interface height, enabling a quantitative comparison between the experiments and simulations. The results for the experiment shown in Figure $3 \mathrm{~b}\left(V_{\mathrm{s}}=5.5 \mu \mathrm{L} ; V_{\mathrm{f}}=6.2 \mu \mathrm{L}\right)$, in which a jet materializes, are given in Figure 4, together with four simulations assuming a small range of prescribed advancing and receding contact angles $\left(\theta_{0}=80^{\circ}\right.$ is fixed) consistent with Figure 2. The inset simulation frames are from the series in Figure $3 \mathrm{~b}\left(\theta_{\mathrm{a}}=105^{\circ} ; \theta_{\mathrm{r}}=50^{\circ}\right)$. Similar features are observed for the four combinations of prescribed contact angles, which cover the spread in the experimental values.

The contact line remains approximately pinned, hence the spread length is constant, between $t=3.0$ and $8.0 \mathrm{~ms}$ in the experiment, demarcated by the vertical dashed lines in Figure 4a. However, once the downward traveling capillary waves reach the contact line at $t \approx 2.0 \mathrm{~ms}$, a reduction in spread length is initiated in the simulations. A decrease in spread length is perhaps counter-intuitive but emphasizes reflection of capillary waves from the contact line and the concurrent upward stretching of the droplet. As noted in the qualitative comparison above, the free surface shapes near the substrate remain comparable between the experiment and simulations throughout, indicating that the apparent contact angles are similar. However, since pinning is not included in the model, the contact line recedes in the simulations. The contact line only pins in the experiment at low contact angles, so there is only a small volume of fluid near the contact line. Hence, the spread length in the simulations recovers to yield excellent agreement with the experiment for $t \geq 8.0 \mathrm{~ms}$, where the spread length quickly increases to its maximum shortly after column collapse has concluded. This agreement indicates that modeling wettability without pinning it is not detrimental to understanding the observed dynamics.

The total droplet height remains approximately constant in Figure $4 \mathrm{~b}$ until the capillary waves arrive at the top of the droplet $(t=3.0 \mathrm{~ms})$, initiating oscillation before stretching the droplet upward to form the column. Due to stretching, the
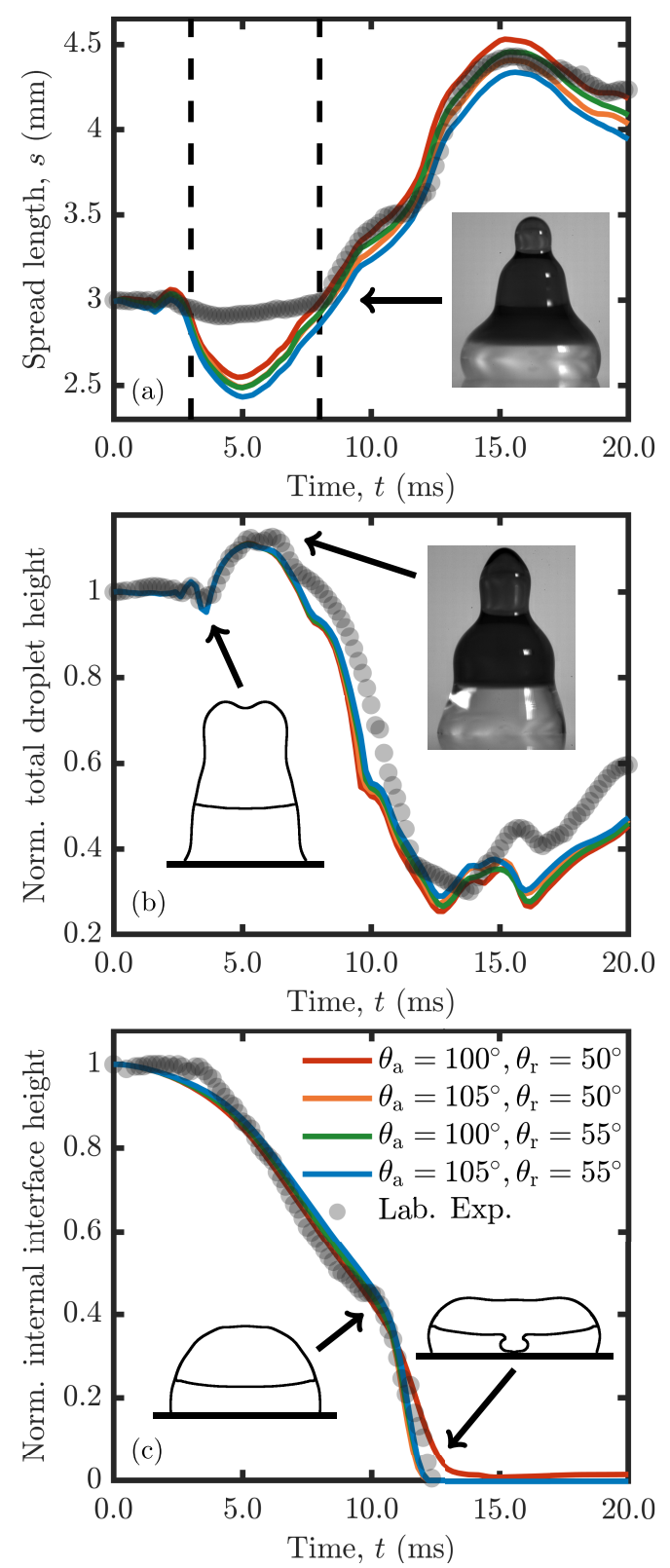

Figure 4. Image-processed experiments with $V_{s}=5.5 \mu \mathrm{L}$ (corresponding to Figure $3 \mathrm{~b}$ ) and simulation results (solid lines). Vertical dashed lines delimit contact line pinning in the experiments. $\theta_{0}=80^{\circ}$ in all simulations. Heights are normalized by their value at $t=$ $0.0 \mathrm{~ms}$.

maximum height attained is significantly greater than at $t=0.0$ $\mathrm{ms}$, before the droplet collapses toward the substrate. The rapid decrease in height is slightly delayed in the experiment compared to the simulations due to the aforementioned differences in spread length. There may also be a small influence here from the slight upward velocity in the experimental initial condition. The rate of collapse temporarily reduces at $t \approx 9.5 \mathrm{~ms}$ in both the experiment and simulations, which coincides with the disappearance of the column, appearing as a shoulder in Figure $4 \mathrm{~b}$. This shoulder is a consequence of contact angle hysteresis delaying outward spreading and has been seen before for similar droplet configurations but higher Ohnesorge number and lower hydrophobicity. ${ }^{43}$ Note that the central depression between $t$ $\approx 11.0$ and $14.0 \mathrm{~ms}$ cannot be perceived from the external side 
view for image processing and so appears as a straight line, whereas a dip is correctly seen in the simulation results. Slightly quicker droplet recoil is seen in the experiment, again due to the differences in spreading. Nevertheless, the same features are evident in both the experiment and simulations, demonstrating that the simulations accurately capture the relevant physics.

Importantly for this study, the internal dynamics are captured in the simulations very well, as demonstrated in Figure $4 \mathrm{c}$ for the internal interface height. The rate and extent of internal interface height decrease is in quantitative agreement with the experiment; internal jet formation is correctly predicted. These observations confirm the applicability of the simulations to study internal jet formation.

Changing Receding Contact Angle. To assess the effect of substrate wettability on jet formation, Figure 5 demonstrates
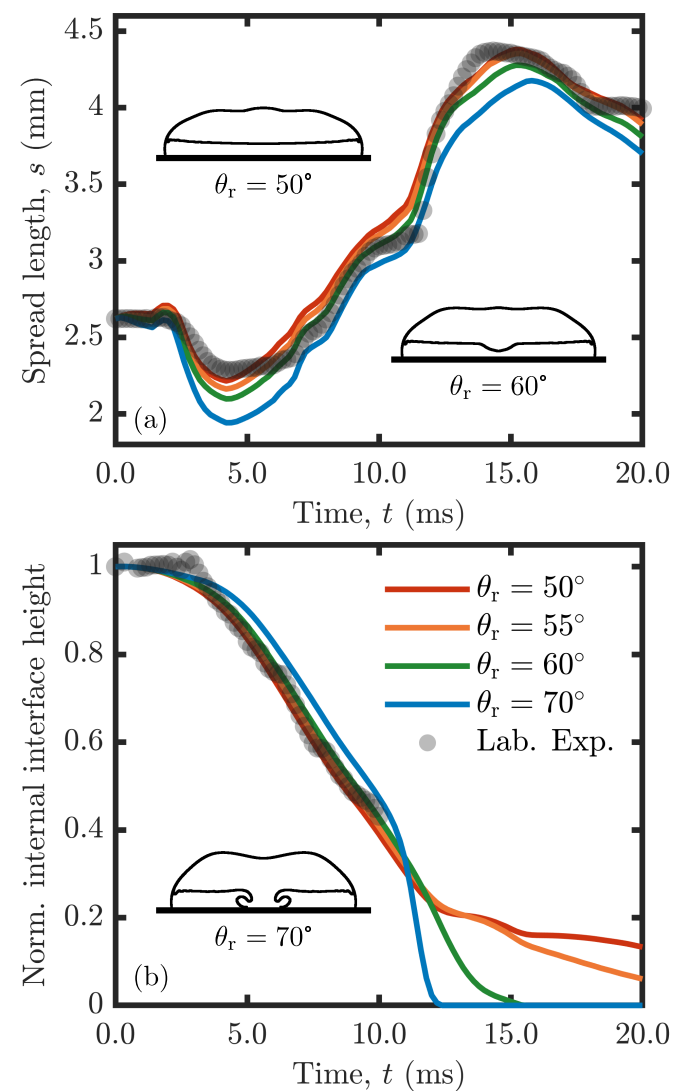

Figure 5. Image-processed experiments with $V_{\mathrm{s}}=3.9 \mu \mathrm{L}$ (corresponding to Figure $3 \mathrm{a}$ ) and simulation results (solid lines). $\theta_{\mathrm{a}}$ $=100^{\circ}$ and $\theta_{0}=82^{\circ}$ in all simulations. Heights are normalized by their value at $t=0.0 \mathrm{~ms}$. The inset frames correspond to $t=13.0 \mathrm{~ms}$.

the consequence of increasing the receding contact angle for the experiment shown in Figure 3a, for which no jet materializes $\left(V_{\mathrm{s}}=3.9 \mu \mathrm{L} ; V_{\mathrm{f}}=6.2 \mu \mathrm{L}\right)$. The image-processed experimental data shows good agreement with the $\theta_{\mathrm{r}}=50^{\circ}$ simulation. $\theta_{\mathrm{a}}=100^{\circ}$ and $\theta_{0}=82^{\circ}$ are fixed.

As the receding contact angle increases, the contact line recedes more freely leading to a greater initial decrease in the spread length, as seen in Figure 5a. With the associated decrease in contact angle hysteresis, the maximum spread length after column collapse also reduces. The shoulder (temporary reduction in the spreading rate) at $t \approx 10.0 \mathrm{~ms}$ also becomes more prominent. These factors combine to hold the

droplet up during the vertical stretching phase of the dynamics (which is also evident in the total droplet height-see the Supporting Information) before column collapse.

The resulting transformation in the internal dynamics seen in Figure $5 \mathrm{~b}$ is more dramatic. While no internal jet is formed with $\theta_{\mathrm{r}}=50^{\circ}$, increasing the receding contact angle (to $\theta_{\mathrm{r}}=$ $70^{\circ}$ ) destabilizes the internal interface to generate a jet, improving advective mixing. The differences in outcome are clear from the inset frames in Figure 5, which show the internal interface and free surface profiles at $t=13.0 \mathrm{~ms}$. Note that the evolution from no jet to jet is not abrupt but occurs over a small range of receding contact angles for which a slow decrease in interface height is seen (e.g., for $\theta_{\mathrm{r}}=60^{\circ}$ ); the internal dynamics are considered to be transitional over this range. A jet is said to have formed only for cases where an abrupt change in the internal interface height is evident (e.g., for $\theta_{\mathrm{r}}=70^{\circ}$ ).

Enhanced vertical stretching on increasing $\theta_{\mathrm{r}}$ yields a greater excess of surface energy to contribute to jet formation. Figure $5 \mathrm{~b}$ also shows that increasing $\theta_{\mathrm{r}}$ holds the internal interface higher during the stretching and initial collapse phases $(t \approx 3.0$ to $10.0 \mathrm{~ms}$ ), which has already been identified above as being beneficial for jet formation. However, the change in substrate wettability also influences the propagation of capillary waves.

Capillary Waves. This section elucidates the free surface dynamics which lead to internal jet formation. Figure 6 shows

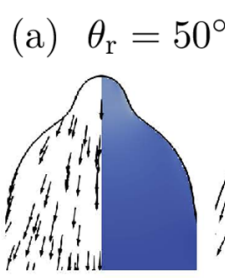

$8.2 \mathrm{~ms}$

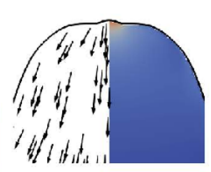

$8.6 \mathrm{~ms}$

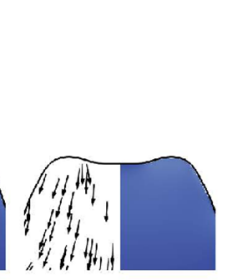

$9.0 \mathrm{~ms}$

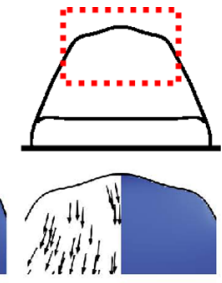

$9.4 \mathrm{~ms}$

(b) $\theta_{\mathrm{r}}=70^{\circ}$

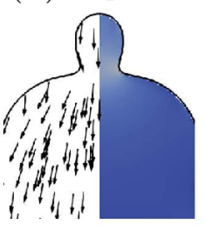

$8.4 \mathrm{~ms}$

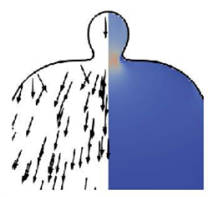

$8.8 \mathrm{~ms}$
0.0 $\left|u_{2}\right|\left(\mathrm{ms}^{-1}\right)$

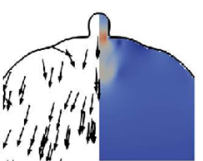

$9.2 \mathrm{~ms}$

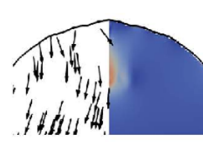

$9.6 \mathrm{~ms}$
Figure 6. Free surface profiles and velocity fields, with $\theta_{\mathrm{a}}=100^{\circ}, \theta_{0}=$ $82^{\circ}, V_{\mathrm{s}}=3.9 \mu \mathrm{L}$, and $V_{\mathrm{f}}=6.2 \mu \mathrm{L}$. The dotted red box on the $9.4 \mathrm{~ms}$ panel (a) frame indicates the extent shown in the other frames. Unscaled velocity vectors (left); contour plot colored by the magnitude of vertical velocity (right). (a) $\theta_{\mathrm{r}}=50^{\circ}$, with no jet. (b) $\theta_{\mathrm{r}}=70^{\circ}$, with a jet.

the free surface profiles and velocity fields at the top of the coalesced droplet, encompassing the end of column collapse for two of the simulations from Figure $5\left(V_{\mathrm{s}}=3.9 \mu \mathrm{L} ; V_{\mathrm{f}}=6.2\right.$ $\mu \mathrm{L} ; \theta_{\mathrm{a}}=100^{\circ} ; \theta_{0}=82^{\circ}$ ). Unscaled velocity vectors are shown on the left; the contour plot on the right is colored by the magnitude of vertical velocity. No jet is seen in Figure 6a, where $\theta_{\mathrm{r}}=50^{\circ}$, while a jet emerges in Figure $6 \mathrm{~b}$, where $\theta_{\mathrm{r}}=$ $70^{\circ}$.

Only in the case with a jet (Figure 6b) does the stretched column thin at its base enough to form a small "tip" on top of the coalesced droplet. The pressure within this tip is elevated due to its high curvature. Together with the constriction at its 
base, a rapid downward vertical flow is thus generated as the tip drains into the bulk, seen in the 8.8 and $9.2 \mathrm{~ms}$ frames of Figure $6 \mathrm{~b}$. This high vertical velocity is maintained in the bulk after the tip has been absorbed and goes on to generate a jet by impingement on the substrate. In contrast, for the case with $\theta_{\mathrm{r}}$ $=50^{\circ}$ (Figure 6a), such a tip is not formed. Hence, there is no means to accelerate the downward vertical flow and a jet does not form. The velocity fields near the substrate (in the undyed fluid region) are similar for both cases and quiescent compared to the tip-generated vertical flow, confirming that the differences in spreading behavior on the momentum in this region do not have a significant role on the large-scale internal dynamics. Jet formation here is therefore associated with thinning of the column at its base to form a neck (thus a tip), which wettability evidently influences.

Such free surface dynamics have been studied in the context of partial coalescence, where the potential for the tip to pinchoff and form a satellite droplet is of interest. A competition between vertical and horizontal collapse owing to surface tension via capillary waves (rather than the Rayleigh-Taylor instability) determines the extent of neck thinning and potential for pinch-off in droplet-pool coalescence. ${ }^{44}$ In free droplet coalescence, it has recently been shown that capillary waves propagating along the larger droplet, in addition to the smaller droplet where column collapse occurs, affect thinning and pinch-off. ${ }^{40}$ Without a substrate, the capillary waves do not typically reach the apex of the larger droplet before pinch-off occurs, ${ }^{27}$ but capillary waves can be reflected away from a substrate and thus influence the dynamics. Note that this work is restricted to cases in which the neck eventually expands to prevent pinch-off; partial coalescence was not seen in the experiments presented. Nevertheless, the influence of capillary waves on neck thinning can be determined from the pressure field in a similar way to the partial coalescence studies.

Figure $7 \mathrm{a}, \mathrm{b}$ shows a contour plot on the right of the droplets, colored by pressure, together with unscaled velocity vectors on the left, for the two cases in Figure 6. Recall that a jet materializes only when $\theta_{\mathrm{r}}=70^{\circ}$ (Figure 6b). The upward traveling capillary waves from meniscus bridge expansion generate the column as previously discussed, while the downward traveling capillary waves are reflected from the substrate at $t \approx 2.0 \mathrm{~ms}$. By $t=4.0 \mathrm{~ms}$, the leading reflected capillary wave is already propagating back up the free surface, seen as a region of positive free surface curvature near the intersection between the internal interface and free surface in Figure $7 \mathrm{a}, \mathrm{b}$. The leading reflected capillary wave is more prominent in Figure $7 \mathrm{~b}$ due to the lower contact angle reduction required for contact line movement (i.e., less energy is dissipated) and the resulting greater reduction in spread length compared to Figure 7a. In each case, the leading reflected capillary wave subsequently interacts with the region of negative curvature at the base of column; the interaction is delineated by the black dotted boxes at $t=5.0 \mathrm{~ms}$. The reflected capillary waves prevent the negative curvature from propagating further down the free surface and dissipating. Instead, the interaction enhances the negative curvature at the base of the column and thus intensifies horizontal collapse. However, the intensity of the low-pressure region behind the negative curvature, and thus the rate of horizontal collapse, depends on the strength of the reflected capillary waves. For the lower receding contact angle (Figure 7a), vertical collapse overcomes the concurrent horizontal collapse before the neck thins enough for a tip to form. For the higher receding contact

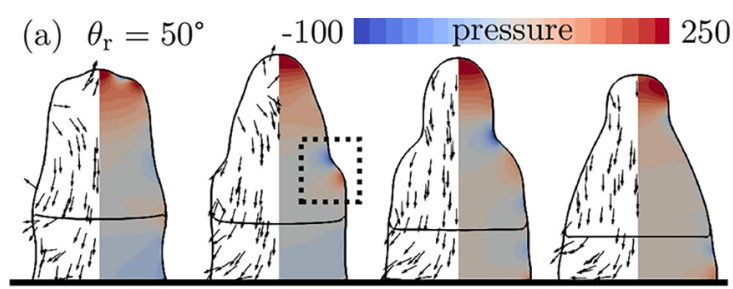

$4.0 \mathrm{~ms}$ $5.0 \mathrm{~ms}$ $6.0 \mathrm{~ms}$ $7.0 \mathrm{~ms}$

\section{(b) $\theta_{\mathrm{r}}=70^{\circ}$}
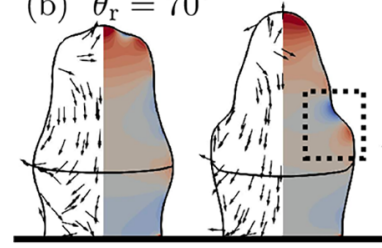

$5.0 \mathrm{~ms}$

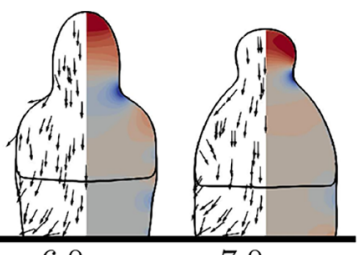

$6.0 \mathrm{~ms}$

$7.0 \mathrm{~ms}$
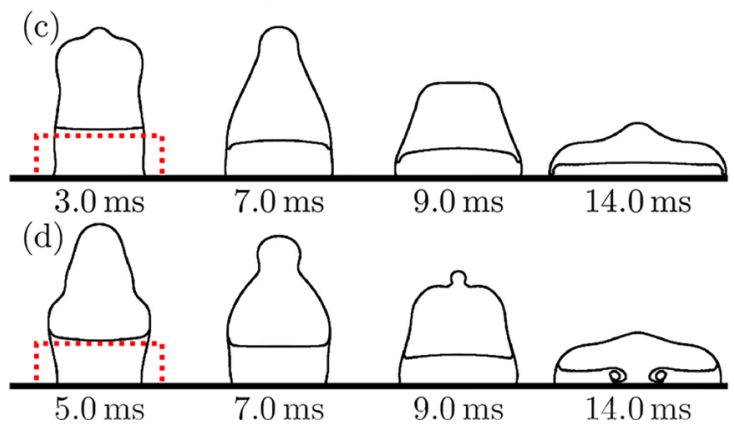

Figure 7. Free surface and internal interface profiles where $\theta_{\mathrm{a}}=100^{\circ}$, $\theta_{0}=82^{\circ}, V_{\mathrm{s}}=3.9 \mu \mathrm{L}$, and $V_{\mathrm{f}}=6.2 \mu \mathrm{L}$. Panels (a) and (b) have unscaled velocity vectors (left) and a contour plot colored by the modified pressure (right). The black dotted boxes highlight capillary wave interactions. (a) $\theta_{\mathrm{r}}=50^{\circ}$, with no jet. (b) $\theta_{\mathrm{r}}=70^{\circ}$, with a jet. Panels (c) and (d) show the same simulation as panel (b), but have their velocities zeroed within $1 \mathrm{~mm}$ of the substrate (delineated by the red dotted boxes) at (c) $t=3.0 \mathrm{~ms}$; (d) $t=5.0 \mathrm{~ms}$.

angle (Figure 7b), an intense low-pressure region is maintained, which leads to sufficient horizontal collapse for a tip to form, though the rate of horizontal collapse is not enough to cause pinch-off. The substrate wettability therefore directly influences the ability to form a tip at the top of the droplet via capillary waves and the ability for a jet to form.

In the context of partial coalescence, several numerical studies have confirmed the influence of capillary waves within a particular region by zeroing the velocity field there after a given time and restarting the simulation. ${ }^{40,44}$ The effect of this process is to nullify the capillary waves in that region. For the case in Figure $7 \mathrm{~b}$ (with $\theta_{\mathrm{r}}=70^{\circ}$ ), in which a jet usually emerges, the simulation was repeated and stopped at $t=3.0$ ms, just after the leading capillary wave had been reflected from the contact line. The velocity field was zeroed within 1 $\mathrm{mm}$ of the substrate (which is below the original sessile droplet height), delineated by the red dotted box in Figure $7 c$, and the simulation restarted. No jet was seen in the restarted simulation, with free surface dynamics similar to Figure $7 \mathrm{a}$ materializing. Consistent with the results above, a tip was not formed.

This zeroing exercise was repeated for various different times; no jet was observed for interruption times of $t=2.0$ and $4.0 \mathrm{~ms}$ either. However, for interruption times of $t=5.0 \mathrm{~ms}$ (shown in Figure 7d) and greater, a jet forms as in the undisturbed simulation. From the free surface profile and pressure distribution of the undisturbed simulation (Figure 
$7 \mathrm{~b})$, the leading reflected capillary wave passes the $1 \mathrm{~mm}$ mark between $t=4.0$ and $5.0 \mathrm{~ms}$, which explains the concurrent transition in jet existence. These tests corroborate the correlation between capillary wave reflection, tip generation, and jet formation.

Droplet Volume and Substrate Wettability. To systematically explore the parameter space, $\theta_{0}=90^{\circ}$ and $V_{\mathrm{f}}$ $=6.0 \mu \mathrm{L}$ are now fixed in the simulations. The receding contact angle, $\theta_{\mathrm{r}}$ and sessile droplet volumne, $V_{\mathrm{s}}$ are varied to produce a regime map for internal jet formation in Figure 8.

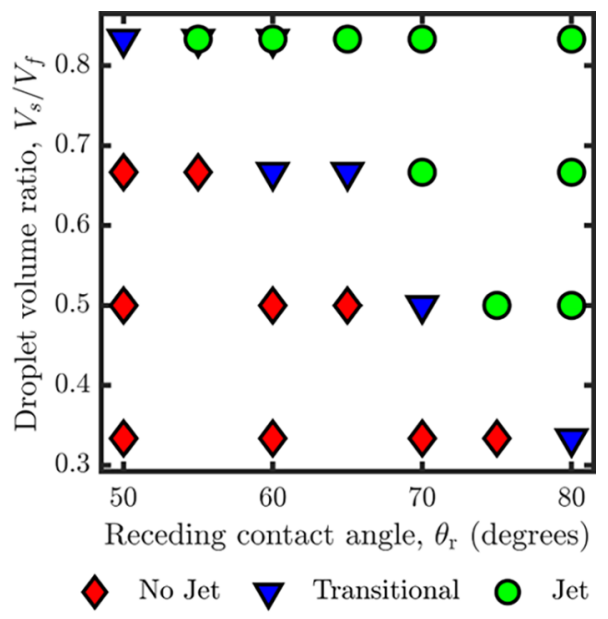

Figure 8. Numerically generated regime map for jet dependence on droplet volume ratio and wettability. For each $\theta_{\mathrm{r}}$ and $V_{\mathrm{s}} / V_{\mathrm{f}}$ combination, three simulations were conducted with $\theta_{\mathrm{a}} \in\{100$, $\left.110,120^{\circ}\right\} . \theta_{0}=90^{\circ}$ and $V_{\mathrm{f}}=6 \mu \mathrm{L}$ are fixed.

Examples of the classifications ("no jet", "transitional" and "jet") are inset in Figure 5. Approximately $2.3 \times 10^{4}$ core hours and 69 simulations were required to produce Figure 8 .

Jet formation is promoted by increasing either the receding contact angle or the (sessile-to-free) droplet volume ratio. For larger volume ratios, the reflected capillary waves must travel further along the originally sessile droplet which reduces their strength due to viscous dissipation. ${ }^{40}$ In fact, there likely exists a critical droplet volume ratio beyond which the substrate does not affect jet formation since the distance that the capillary waves must travel before having any effect is too far. In this case, the internal dynamics are likely to be akin to those of free droplets, especially in regard to the mechanisms underpinning jet formation. However, the effect of the substrate will be felt for droplets of small and similar volume ratios which are of greatest practical (e.g., lab-on-a-chip and inkjet) and fundamental interest. For a given sessile droplet volume, decreasing the equilibrium contact angle, $\theta_{0}$ yields greater curvature within the meniscus bridge and stronger capillary waves. ${ }^{40}$ Conversely, increasing free surface area escalates viscous dissipation.

The advancing contact angle, $\theta_{\mathrm{a}}$ should also be considered. In fact, for each $\left\{V_{\mathrm{s}} / V_{\mathrm{f}}, \theta_{\mathrm{r}}\right\}$ combination in Figure 8, three simulations with different advancing contact angles, $\theta_{\mathrm{a}} \in\{100$, $\left.110,120^{\circ}\right\}$ were conducted and are plotted. No differences in classification transpired, except for the largest sessile droplet volume studied $\left(V_{\mathrm{s}}=5.0 \mu \mathrm{L}\right)$ when $\theta_{\mathrm{r}}=55^{\circ}$ or $\theta_{\mathrm{r}}=60^{\circ}$, for which an advancing contact angle of $\theta_{\mathrm{a}}=120^{\circ}$ incited jet formation (as opposed to the transitional situation for $\theta_{\mathrm{a}} \in$ $\left.\left\{100,110^{\circ}\right\}\right)$. An increase in $\theta_{\mathrm{a}}$ restricts outward spreading which may be somewhat beneficial for jet formation by increasing the internal interface height and concentrating the momentum generated by the tip. However, the influence of the advancing contact angle is subordinated to that of the receding contact angle due to the latter's influence on reflection of the leading capillary wave. Hence, jet formation in this configuration is only weakly dependent on the advancing contact angle.

For a fixed advancing contact angle, modifying the receding contact angle also adjusts the contact angle hysteresis. Previous work has indicated that low hysteresis is beneficial for mixing. ${ }^{9}$ Whilst this inference is generally supported by the current work, each point in Figure 8 contains a $20^{\circ}$ degree hysteresis range which indicates that hysteresis is not the decisive factor in jet formation. Furthermore, replotting Figure 8 against the hysteresis, $\theta_{\mathrm{a}}-\theta_{\mathrm{r}}$ instead of $\theta_{\mathrm{r}}$ (see Supporting Information) shows an acute lack of clear regime boundaries, demonstrating that hysteresis itself is not an accurate predictor of jet formation.

For $V_{\mathrm{s}}=3.0 \mu \mathrm{L}$ and $\theta_{0}=90^{\circ}$, the sessile droplet is exactly half of the free droplet $\left(V_{\mathrm{f}}=6.0 \mu \mathrm{L}\right)$. Hence, the initial droplet curvatures and Laplace pressures are identical, yet an internal jet can still form if $\theta_{\mathrm{r}} \geq 75^{\circ}$. Moreover, the Laplace pressure of a $2.0 \mu \mathrm{L}$ sessile droplet (with $\theta_{0}=90^{\circ}$ ) is greater than that of a $6.0 \mu \mathrm{L}$ free droplet, whilst the latter is injected into the former, opposing the initial Laplace pressure difference. Coalescence in this configuration therefore provides the ability to mix droplets in unconventional ways, without relying on a particular Laplace pressure difference. Furthermore, Figure 8 indicates that internal jet formation can be expected for a wide range of substrate wettabilities when the initial droplet Laplace pressures are equal, unlike in free droplet coalescence, which may be desirable in microfluidic applications.

Droplet Viscosity. It is well known that increasing viscosity increases viscous dissipation, which dampens capillary waves and suppresses internal flows. However, increasing viscosity also reduces the rate of change of curvature during meniscus bridge expansion and so reduces the strength of emitted capillary waves. ${ }^{45}$ Column formation and the strength of reflected capillary waves, both of which have been shown to be beneficial for jet formation already in this work, are therefore diminished with increasing droplet viscosity. Hence, it is expected that increasing droplet viscosity should be unfavorable for jet formation. This prediction is confirmed in Figure 9 for which $\theta_{\mathrm{a}}=100^{\circ}, \theta_{0}=90^{\circ}, V_{\mathrm{s}}=4.0 \mu \mathrm{L}$, and $V_{\mathrm{f}}=$ $6.0 \mu \mathrm{L}$ (corresponding to the second highest row in Figure 8); the droplet viscosity $\mu_{\mathrm{d}}$ and receding contact angle $\theta_{\mathrm{r}}$ are varied. The same trend in jet formation with respect to the receding contact angle identified above is seen across different droplet viscosities. Indeed, the existence of an internal jet in Figure 9 exactly coincides with the presence of a tip of high curvature identified as being crucial for internal jet formation in previous sections for a fixed droplet viscosity. The key result is thus confirmed across a range of droplet viscosities. Interestingly, the extent of the transitional viscosity range is similar across those receding contact angles exhibiting all three regimes.

The droplet viscosity affects the dynamics both via the Ohnesorge number, determining the degree of surface tension dominance, and the droplet/outer phase viscosity ratio. Changes in either viscosity affect capillary wave propagation, ${ }^{40}$ while only the droplet viscosity feeds into the Ohnesorge number as defined above. Consideration is limited to exploring 


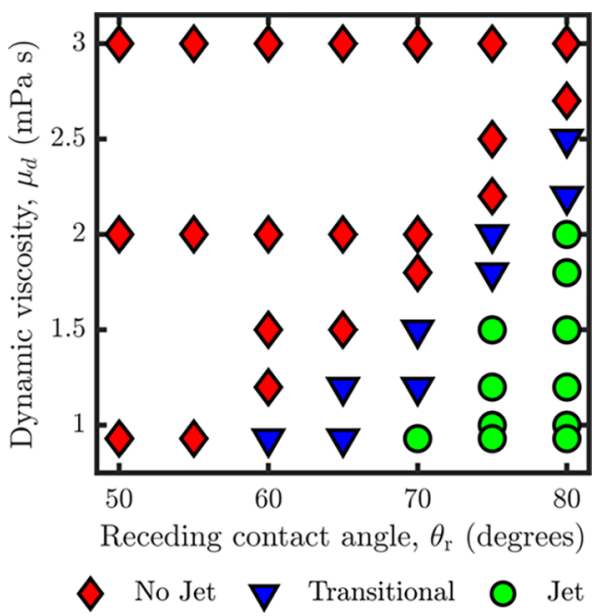

Figure 9. Numerically generated regime map for jet dependence on droplet viscosity and receding contact angle. $\theta_{\mathrm{a}}=100^{\circ}, \theta_{0}=90^{\circ}, V_{\mathrm{s}}=$ $4.0 \mu \mathrm{L}$, and $V_{\mathrm{f}}=6.0 \mu \mathrm{L}$ in all simulations.

droplet viscosity changes here with respect to likely applications. It is important to appreciate though that direct extrapolation to changes in the Ohnesorge number or viscosity ratio is not appropriate. Hence, the data in Figure 9 are plotted against droplet viscosity $\mu_{\mathrm{d}}$ rather than the viscosity ratio or Ohnesorge number.

According to Figure 9, a jet can be formed when $\theta_{\mathrm{r}}=80^{\circ}$ and $\mathrm{Oh}=7.0 \times 10^{-3}$ (based on $\mu_{\mathrm{d}}=2.0 \mathrm{mPa} \mathrm{s}$ ), given a volume ratio of $V_{\mathrm{s}} / V_{\mathrm{f}}=0.667$. This volume ratio is remarkably small compared to what would be required in free droplets. As explained above, the free droplet is injected into the sessile droplet and so assumes the same role as the smaller droplet in free droplet coalescence, regardless of the relative droplet volumes. Thus, taking the free droplet radius $\left(r_{\mathrm{f}}=1.127 \mathrm{~mm}\right)$ as the length scale, a (linear) size ratio of 1.23 would be required to form a jet at the equivalent Ohnesorge number in free droplet coalescence according to a recent work. ${ }^{15}$ This size ratio corresponds to a volume ratio of 1.88 , almost a 3 -fold increase on the value identified above. Considering the effective radius of the sessile droplet (i.e., the radius of the sphere of which the spherical cap is part) at the onset of coalescence, the effective size ratio between the droplets is 1.10. Nevertheless, it is clear that the substrate enhances jet formation here and there is a potential to enable jet formation with larger droplet viscosities compared to free droplet coalescence.

\section{CONCLUSIONS}

This work has explored internal jet formation during the coalescence of an initially static free droplet with a sessile droplet of the same fluid, focusing on the influence of substrate wettability. The dynamics were successfully simulated using a VOF model, showing good quantitative agreement with the laboratory experiments, which are reported too. The use of the Kistler dynamic contact angle model allowed the substrate wettability to be captured very accurately despite the model not including pinning.

Compared to free droplet coalescence, where a jet may be formed when the smaller droplet is drawn directly into the larger one, on a substrate an internal jet can arise via a different mechanism. Here, jet formation was shown to depend on a thinning neck that led to a tip with high curvature and pressure on top of the coalesced droplet, which incited a rapid downward flow toward the substrate. The generation of this tip depends on the contact line dynamics via reflected capillary waves. Substrate wettability therefore directly influences jet formation and mixing efficiency. Strong reflection of capillary waves is required to generate sufficient neck thinning, so higher receding contact angles are beneficial for producing a jet.

The ability to assemble a regime map of the droplet volume ratio (sessile-to-free) against the receding contact angle, with clear regime boundaries, demonstrates the influence of substrate wettability and confirms that increasing the volume ratio encourages jet formation, as expected. However, the reflected capillary wave mechanism identified in this work can lead to jet formation at very different volume ratios from those that produce jets during free droplet coalescence. While it is generally true that low contact angle hysteresis is beneficial for mixing, hysteresis itself is not an accurate predictor of jet formation; the effect of the advancing contact angle is subordinated to that of the receding contact angle. Jet formation is inhibited by increasing droplet viscosity, though jets can appear at droplet viscosities higher than expected for free droplets for a given volume ratio.

The results indicate the potential of engineering substrate wettability to improve mixing via the formation of internal jets in microfluidic systems. To achieve good advective mixing, capillary waves reflected from the contact line should be enhanced where possible, which is achieved by increasing the receding contact angle in the configuration studied. The influence of contact line movement in a certain direction may be dominant, so contact angle hysteresis alone is not necessarily the decisive factor. It is therefore imperative to consider substrate wettability for applications involving coalescing droplets for which the internal dynamics, and associated fluid mixing, may be influential.

\section{ASSOCIATED CONTENT}

\section{SI Supporting Information}

The Supporting Information is available free of charge at https://pubs.acs.org/doi/10.1021/acs.langmuir.0c01689.

Supplementary graphs and numerical solver details (including discretization schemes, linear solvers, and explanation of the compression velocity); Laplace pressure recovery; Mesh sensitivity analysis; Interface compression velocity; Solution and algorithm control; Analytical droplet pressure difference across the free surface compared to the simulated results for a free droplet of radius (Figure S1); Mesh details for the mesh sensitivity analysis (Table S1) (PDF)

Video of the experiment in Figure 3a (MP4)

Video of the simulation in Figure 3a (MP4)

Video of the experiment in Figure 3b (MP4)

Video of the simulation in Figure $3 \mathrm{~b}$ (MP4)

Video of the experiment in Figure 3c (MP4)

Video of the simulation in Figure 7a (MP4)

Video of the simulation in Figure $7 \mathrm{~b}$ (MP4)

\section{AUTHOR INFORMATION}

\section{Corresponding Authors}

Thomas C. Sykes - EPSRC Centre for Doctoral Training in Fluid Dynamics, University of Leeds, Leeds LS2 9JT, United Kingdom; 이이이.org/0000-0002-9996-3004;

Email: t.c.sykes@outlook.com 
Mark C. T. Wilson - School of Mechanical Engineering, University of Leeds, Leeds LS2 9JT, United Kingdom; ○ orcid.org/0000-0002-1058-2003; Email: m.wilson@ leeds.ac.uk

\section{Authors}

David Harbottle - School of Chemical and Process Engineering, University of Leeds, Leeds LS2 9JT, United Kingdom; (1) orcid.org/0000-0002-0169-517X

Zinedine Khatir - School of Engineering and the Built Environment, Birmingham City University, Birmingham B4 $7 X G$, United Kingdom

Harvey M. Thompson - School of Mechanical Engineering, University of Leeds, Leeds LS2 9JT, United Kingdom

Complete contact information is available at:

https://pubs.acs.org/10.1021/acs.langmuir.0c01689

\section{Notes}

The authors declare no competing financial interest.

\section{ACKNOWLEDGMENTS}

The authors thank Karrar H. Al-Dirawi, Khaled H. A. AlGhaithi, Oliver G. Harlen, Chris S. Hodges (all from the University of Leeds), and Suparit Tangparitkul (Chiang Mai University) for insightful discussions and helpful suggestions. This work was generously supported by the Engineering and Physical Sciences Research Council (EPSRC) Centre for Doctoral Training in Fluid Dynamics at the University of Leeds [grant number EP/L01615X/1]. The numerical work was undertaken on ARC2, ARC3, and ARC4, all part of the High Performance Computing facilities at the University of Leeds, U.K.

\section{REFERENCES}

(1) Sellier, M.; Trelluyer, E. Modeling the coalescence of sessile droplets. Biomicrofluidics 2009, 3, No. 022412.

(2) Lee, M. W.; Kang, D. K.; Yoon, S. S.; Yarin, A. L. Coalescence of two drops on partially wettable substrates. Langmuir 2012, 28, 37913798.

(3) Blanchette, F. Simulation of mixing within drops due to surface tension variations. Phys. Rev. Lett. 2010, 105, No. 074501.

(4) Yeh, S.-I.; Fang, W.-F.; Sheen, H.-J.; Yang, J.-T. Droplets coalescence and mixing with identical and distinct surface tension on a wettability gradient surface. Microfluid. Nanofluid. 2013, 14, 785795.

(5) Stone, H. A.; Stroock, A. D.; Ajdari, A. Engineering flows in small devices: microfluidics toward a lab-on-a-chip. Annu. Rev. Fluid Mech. 2004, 36, 381-411.

(6) Kovalchuk, N. M.; Reichow, M.; Frommweiler, T.; Vigolo, D.; Simmons, M. J. H. Mass transfer accompanying coalescence of surfactant-laden and surfactant-free drop in a microfluidic channel. Langmuir 2019, 35, 9184-9193.

(7) Sykes, T. C.; Castrejón-Pita, A. A.; Castrejón-Pita, J. R.; Harbottle, D.; Khatir, Z.; Thompson, H. M.; Wilson, M. C. T. Surface jets and internal mixing during the coalescence of impacting and sessile droplets. Phys. Rev. Fluids 2020, 5, No. 023602.

(8) Jehannin, M.; Charton, S.; Karpitschka, S.; Zemb, T.; Möhwald, H.; Riegler, H. Periodic precipitation patterns during coalescence of reacting sessile droplets. Langmuir 2015, 31, 11484-11490.

(9) Nilsson, M. A.; Rothstein, J. P. The effect of contact angle hysteresis on droplet coalescence and mixing. J. Colloid Interface Sci. 2011, 363, 646-654.

(10) Lai, Y.-H.; Hsu, M.-H.; Yang, J.-T. Enhanced mixing of droplets during coalescence on a surface with a wettability gradient. Lab Chip 2010, 10, 3149-3156.
(11) Anilkumar, A. V.; Lee, C. P.; Wang, T. G. Surface-tensioninduced mixing following coalescence of initially stationary drops. Phys. Fluids A 1991, 3, 2587-2591.

(12) Eiswirth, R. T.; Bart, H.-J.; Ganguli, A. A.; Kenig, E. Y. Experimental and numerical investigation of binary coalescence: Liquid bridge building and internal flow fields. Phys. Fluids 2012, 24, No. 062108.

(13) Liu, D.; Zhang, P.; Law, C. K.; Guo, Y. Collision dynamics and mixing of unequal-size droplets. Int. J. Heat Mass Transfer 2013, 57, 421-428.

(14) Nobari, M. R.; Tryggvason, G. The Flow Induced by the Coalescence of Two Initially Stationary Drops; NASA Technical Memorandum: Ann Arbor, MI, 1994; p 106752.

(15) Xia, X.; He, C.; Yu, D.; Zhao, J.; Zhang, P. Vortex-ring-induced internal mixing upon the coalescence of initially stationary droplets. Phys. Rev. Fluids 2017, 2, No. 113607.

(16) Tang, C.; Zhao, J.; Zhang, P.; Law, C. K.; Huang, Z. Dynamics of internal jets in the merging of two droplets of unequal sizes. J. Fluid Mech. 2016, 795, 671-689.

(17) Saha, A.; Wei, Y.; Tang, X.; Law, C. K. Kinematics of vortex ring generated by a drop upon impacting a liquid pool. J. Fluid Mech. 2019, 875, 842-853.

(18) Nowak, E.; Xie, Z.; Kovalchuk, N. M.; Matar, O. K.; Simmons, M. J. H. Bulk advection and interfacial flows in the binary coalescence of surfactant-laden and surfactant-free drops. Soft Matter 2017, 13, $4616-4628$.

(19) Luo, X.; Yin, H.; Ren, J.; Yan, H.; Huang, X.; Yang, D.; He, L. Enhanced mixing of binary droplets induced by capillary pressure. $J$. Colloid Interface Sci. 2019, 545, 35-42.

(20) Castrejón-Pita, J. R.; Kubiak, K. J.; Castrejón-Pita, A. A.; Wilson, M. C. T.; Hutchings, I. M. Mixing and internal dynamics of droplets impacting and coalescing on a solid surface. Phys. Rev. E 2013, 88, No. 023023 .

(21) Narhe, R.; Beysens, D.; Nikolayev, V. S. Contact line dynamics in drop coalescence and spreading. Langmuir 2004, 20, 1213-1221.

(22) Raman, K. A. Dynamics of simultaneously impinging drops on a dry surface: Role of inhomogeneous wettability and impact shape. J. Colloid Interface Sci. 2018, 516, 232-247.

(23) Raman, K. A.; Jaiman, R. K.; Lee, T.-S.; Low, H.-T. Lattice Boltzmann study on the dynamics of successive droplets impact on a solid surface. Chem. Eng. Sci. 2016, 145, 181-195.

(24) Quetzeri-Santiago, M. A.; Yokoi, K.; Castrejón-Pita, A. A.; Castrejón-Pita, J. R. Role of the dynamic contact angle on splashing. Phys. Rev. Lett. 2019, 122, No. 228001.

(25) Al-Dirawi, K. H.; Bayly, A. E. A new model for the bouncing regime boundary in binary droplet collisions. Phys. Fluids 2019, 31, No. 027105.

(26) Berry, J. D.; Dagastine, R. R. Mapping coalescence of micronsized drops and bubbles. J. Colloid Interface Sci. 2017, 487, 513-522.

(27) Zhang, F. H.; Li, E. Q.; Thoroddsen, S. T. Satellite formation during coalescence of unequal size drops. Phys. Rev. Lett. 2009, 102, No. 104502.

(28) Hirt, C. W.; Nichols, B. D. Volume of fluid (VOF) method for the dynamics of free boundaries. J. Comput. Phys. 1981, 39, 201-225.

(29) Brackbill, J. U.; Kothe, D. B.; Zemach, C. A continuum method for modeling surface tension. J. Comput. Phys. 1992, 100, 335-354.

(30) Rusche, H. Computational Fluid Dynamics of Dispersed TwoPhase Flows at High Phase Fractions. Ph.D. Thesis, Imperial College, University of London, 2002.

(31) Malgarinos, I.; Nikolopoulos, N.; Marengo, M.; Antonini, C.; Gavaises, M. VOF simulations of the contact angle dynamics during the drop spreading: Standard models and a new wetting force model. Adv. Colloid Interface Sci. 2014, 212, 1-20.

(32) Kistler, S. F. Wettability; Berg, J. C., Ed.; Dekker: New York, 1993; pp 311-429.

(33) Hoffman, R. L. A study of the advancing interface. I. Interface shape in liquid-gas systems. J. Colloid Interface Sci. 1975, 50, 228-241. 
(34) Roisman, I. V.; Rioboo, R.; Tropea, C. Normal impact of a liquid drop on a dry surface: model for spreading and receding. Proc. R. Soc. London, Ser. A 2002, 458, 1411-1430.

(35) Farhangi, M. M.; Graham, P. J.; Choudhury, N. R.; Dolatabadi, A. Induced detachment of coalescing droplets on superhydrophobic surfaces. Langmuir 2012, 28, 1290-1303.

(36) Chu, F.; Li, S.; Ni, Z.; Wen, D. Departure velocity of rolling droplet jumping. Langmuir 2020, 36, 3713-3719.

(37) Rattner, A. S.; Garimella, S. Simple mechanistically consistent formulation for volume-of-fluid based computations of condensing flows. J. Heat Transfer 2014, 136, No. 071501.

(38) Nabil, M.; Rattner, A. S. interThermalPhaseChangeFoam - A framework for two-phase flow simulations with thermally driven phase change. SoftwareX 2016, 5, 216-226.

(39) Wasserfall, J.; Figueiredo, P.; Kneer, R.; Rohlfs, W.; Pischke, P. Coalescence-induced droplet jumping on superhydrophobic surfaces: Effects of droplet mismatch. Phys. Rev. Fluids 2017, 2, No. 123601.

(40) Deka, H.; Biswas, G.; Chakraborty, S.; Dalal, A. Coalescence dynamics of unequal sized drops. Phys. Fluids 2019, 31, No. 012105.

(41) Boelens, A.; Latka, A.; de Pablo, J. Observation of the pressure effect in simulations of droplets splashing on a dry surface. Phys. Rev. Fluids 2018, 3, No. 063602.

(42) Mirzaei, M. A new method for measuring the contact angles from digital images of liquid drops. Micron 2017, 102, 65-72.

(43) Wang, L.; Sun, J. Lattice Boltzmann modeling for the coalescence between a free droplet in gases and a sessile droplet on wettable substrate with contact angle hysteresis. Proc. Inst. Mech. Eng., Part C 2018, 232, 431-444.

(44) Blanchette, F.; Bigioni, T. P. Partial coalescence of drops at liquid interfaces. Nat. Phys. 2006, 2, 254-257.

(45) Thoroddsen, S. T.; Takehara, K.; Etoh, T. G. The coalescence speed of a pendent and a sessile drop. J. Fluid Mech. 2005, 527, 85114. 